\title{
Lack of inducible nitric oxide synthases attenuates leukocyte-endothelial cell interactions in retinal microcirculation
}

\author{
D Iwama, ${ }^{1}$ S Miyahara, ${ }^{2}$ H Tamura, ${ }^{1}$ K Miyamoto, ${ }^{1}$ F Hirose, ${ }^{1}$ N Yoshimura ${ }^{1}$
}

${ }^{1}$ Department of Ophthalmology and Visual Sciences, Kyoto University Graduate School of Medicine, Kyoto, Japan;

${ }^{2}$ Department of Ophthalmology, Otsu Red Cross Hospital, Otsu, Japan

Correspondence to: Dr H Tamura, Department of Ophthalmology and Visual Sciences, Kyoto University Graduate School of Medicine, Kyoto, 606-8507, Japan; htamura-kyt@umin.ac.jp

Accepted 8 February 2008

\section{ABSTRACT}

Aim: To investigate the effect of inducible nitric oxide synthases (iNOS) on inflammatory reactions during endotoxin-induced uveitis (EIU) in mice by studying leukocyte-endothelial cell interactions.

Methods: EIU was produced in immunosuppressed iNOS $^{-1-}$ mice and C57BL/6 (normal) mice by footpad injection of lipopolysaccharide. Leukocytes were labelled with acridine orange. Leukocyte rolling in the retinal microcirculation was evaluated in vivo with acridine orange digital fluorography. The number of migrated leukocytes was counted in flat-mounted retina.

Results: Both leukocyte rolling and migration peaked at $48 \mathrm{~h}$ after lipopolysaccharide injection. The maximal numbers of rolling leukocytes in the immunosuppressed iNOS $^{-1-}$ mouse retina decreased by 98.2\% $(p<0.001)$ compared with that in the normal mouse retina at $48 \mathrm{~h}$ after lipopolysaccharide injection. In addition, the maximal numbers of migrated leukocytes in the immunosuppressed iNOS $^{-1-}$ mouse retina decreased by $74.0 \%$ $(p<0.001)$ compared with that in the normal mouse retina at $24 \mathrm{~h}$ after lipopolysaccharide injection.

Furthermore, the diameters of major retinal veins of the immunosuppressed iNOS $^{-1-}$ group were smaller at both 24 and $48 \mathrm{~h}$ after lipopolysaccharide injection than were those of the normal group ( $p<0.001$, respectively). Conclusions: A lack of iNOS suppresses leukocyteendothelial cell interactions in the retinas of mice with EIU. This suggests that iNOS may play a role in the management of patients with uveitis and other inflammatory conditions.

Leukocytes play a crucial role in inflammatory conditions by interacting with endothelial cells and migrating to the site of inflammation. In the inflamed area, endothelial cells are activated to express adhesion molecules that cause leukocyteendothelial cell interactions through a multistep process. ${ }^{12}$ Initially, leukocytes interact with Pselectin, which is expressed on endothelial cells, and begin rolling along vessel walls. The leukocytes then interact with intercellular adhesion molecule 1 (ICAM-1), adhere to endothelial cells and migrate out of the vessels. These leukocytes subsequently release cytokines and produce proteases and superoxide radical species, which also participate in the cascade of inflammation. ${ }^{3}{ }^{4}$ Because the reactions of these leukocytes cause inflammatory tissue injury or endothelial cell injury, ${ }^{5}$ it is important to evaluate the behaviour of leukocytes in vivo.

Endotoxin-induced uveitis (EIU) is an ocular inflammation model created in an experimental animal by a subcutaneous injection of lipopolysaccharide, ${ }^{7}$ without direct exposure to the eye. ${ }^{89}$ EIU is characterised by leukocyte infiltration with blood-ocular barrier disruption. In this model, inflammatory reactions have been reported to occur in both the anterior and posterior segments of the eye, including infiltration of leukocytes into the aqueous humour, vitreous cavity and retina..$^{8-10}$

During EIU, the expression of inducible nitric oxide synthase (iNOS) is upregulated and is thought to play a key role in the pathogenesis of EIU. ${ }^{11}$ Nitric oxide (NO), derived from iNOS, regulates the expression of P-selectin and ICAM-1 and contributes to vasodilation. ${ }^{12-14}$ In addition, iNOS expression in the retina is likely to cause tissue damage by interfering with the beneficial activities of constitutive neuronal nitric oxide synthase (NOS) and endothelial NOS. ${ }^{15-17}$ Little is known, however, regarding the exact relationship between iNOS and tissue damage.

In-vivo methods to evaluate quantitatively leukocyte-endothelial cell interactions have been established in mouse retina. ${ }^{18}$ Using these methods, we can physiologically evaluate the number of rolling leukocytes and the number of migrated leukocytes in mouse retina. With the recent progress in gene technology, represented by various knockout or transgenic mouse strains, the expression of specific genes can now be easily modified. Accordingly, in-vivo observations of leukocyte rolling and migration in mouse retina now accurately evaluate the genetic regulation of leukocyte behaviour in the inflammatory response.

In the study described here, we quantitatively evaluated the influence of iNOS on leukocyteendothelial cell interactions in the lipopolysaccharide-stimulated retina with the use of mice lacking iNOS. ${ }^{19}$

\section{METHODS}

\section{Animal model}

All experiments were performed in accordance with the ARVO Statement for the Use of Animals in Ophthalmic and Vision Research. Male pigmented C57BL/6J Jms Slc mice (normal group, 8-10 weeks old; $\mathrm{n}=84$ ) were obtained from Japan SLC, Inc (Shizuoka, Japan); male immunosuppressed $\mathrm{iNOS}^{-1-}$ mice (iNOS ${ }^{-}$group, 8-10 weeks old; $\mathrm{n}=84$ ) were obtained from the Jackson Laboratory (Bar Harbor, Maine, USA). EIU was produced in mice by injecting $100 \mu \mathrm{g}$ lipopolysaccharide (Salmonella typhimurium; Sigma Chemical Co, St Louis, Missouri, USA) diluted in $0.1 \mathrm{ml}$ sterile saline into one hind footpad of each animal. Control mice received a footpad injection 
Table 1 Physiological variables

\begin{tabular}{|c|c|c|c|c|c|c|c|}
\hline & $\begin{array}{l}\text { Control } \\
\text { (SEM) }\end{array}$ & $\begin{array}{l}4 \mathrm{~h} \\
\text { (SEM) }\end{array}$ & $\begin{array}{l}12 \mathrm{~h} \\
\text { (SEM) }\end{array}$ & $\begin{array}{l}24 \mathrm{~h} \\
\text { (SEM) }\end{array}$ & $\begin{array}{l}48 \mathrm{~h} \\
\text { (SEM) }\end{array}$ & $\begin{array}{l}72 \mathrm{~h} \\
\text { (SEM) }\end{array}$ & $\begin{array}{l}96 \mathrm{~h} \\
\text { (SEM) }\end{array}$ \\
\hline \multicolumn{8}{|l|}{ Normal } \\
\hline WBC, $\times 10^{3} / \mu \mathrm{l}$ & $8.6(0.8)$ & $7.8(1.0)$ & $7.3(0.9)$ & $7.7(1.9)$ & $13.3(1.4)^{*}$ & $10.9(1.1)$ & $9.4(1.1)$ \\
\hline Heart rate, beats/min & $287(6)$ & $287(8)$ & $282(6)$ & $285(8)$ & $287(8)$ & $282(6)$ & $283(6)$ \\
\hline \multicolumn{8}{|l|}{ iNOS knockout } \\
\hline WBC, $\times 10^{3} / \mu \mathrm{l}$ & $8.1(0.3)$ & $6.7(1.3)$ & $7.4(0.8)$ & $7.7(0.5)$ & $11.1(0.5)^{*}$ & $9.6(0.9)$ & $8.7(1.2)$ \\
\hline
\end{tabular}

iNOS, inducible nitric oxide synthase; MABP, mean arterial blood pressure; WBC, white blood cell peripheral leukocyte count.

Values are means (SEM). $n=6$ at each timepoint in both groups.

There were no significant differences between the normal and iNOS knockout mice group.

${ }^{*} p<0.05$ compared with control values in each group.

of saline alone. All mice were maintained in an air-conditioned room with a 12-h light/12-h dark cycle and given free access to water and food until they were used for the experiments.

\section{Leukocyte rolling in mouse retina}

To evaluate leukocyte rolling during an episode of EIU in mouse retina, we used acridine orange (AO) digital fluorography, a method that has been used to evaluate leukocyte-endothelial cell interactions in mouse retina, as previously described. ${ }^{18}$ In brief, a scanning laser ophthalmoscope (Rodenstock Instruments, Munich, Germany), coupled with a computerassisted image analysis system (Radius, San Jose, California, USA), made continuous high-resolution images of the fundus, which had been stained by metachromatic fluorochrome AO (Wako Pure Chemicals, Osaka, Japan), which emits a green fluorescence when it interacts with DNA. An argon blue laser was used as the illumination source, with a regular emission filter for fluorescein angiography, because the spectral properties of leukocytes stained with $\mathrm{AO}$ are similar to those stained with sodium fluorescein. For further analysis, the images obtained were recorded on an S-VHS videotape at the rate of 30 frames per second.

AO digital fluorography was performed at 4, 12, 24, 48, 72 and $96 \mathrm{~h}$ after lipopolysaccharide injection. Six different mice from each group were used at each timepoint.

Immediately before $\mathrm{AO}$ digital fluorography, the mice were anaesthetised with xylazine hydrochloride $(4 \mathrm{mg} / \mathrm{kg})$ and ketamine hydrochloride $(10 \mathrm{mg} / \mathrm{kg})$ and their pupils were dilated with $0.5 \%$ tropicamide and $2.5 \%$ phenylephrine hydrochloride. Body temperature was maintained at between $37^{\circ} \mathrm{C}$ and $39^{\circ} \mathrm{C}$ throughout the experiment. A contact lens was used to retain corneal clarity throughout the experiment. Each mouse had a catheter inserted into the femoral vein and was placed on a moveable platform. $\mathrm{AO}(0.01 \%$ solution in saline) was injected continuously through the catheter for 1 minute at a rate of $1 \mathrm{ml} /$ minute. Rolling leukocytes were defined as leukocytes that moved at a velocity slower than that of free-flowing leukocytes. The number of rolling leukocytes was calculated from the number of cells per minute crossing a fixed area of the vessel at a distance two disc diameters from the optic disc centre. The flux of rolling leukocytes for each mouse was defined as the average of individual numbers of rolling leukocytes seen in all major veins.

The diameters of the major retinal vessels were measured at two disc diameters from the centre of the optic disc in monochromatic images recorded before AO injection. Each vessel diameter was calculated in pixels as the distance between the half-height points determined separately on each side of the density profile of the vessel image and converted into real values using the calibration factor. The averages of the individual arterial and venous diameters were used as the arterial and venous diameters for each mouse.

\section{Number of leukocytes migrating in mouse retina}

The number of leukocytes migrating in mouse retina was evaluated in flat-mounted retina after $\mathrm{AO}$ digital fluorography. Six different mice from each group were used at each timepoint. Thirty minutes after the injection of $\mathrm{AO}$, one eye from each of six mice was enucleated. Blood was collected to count the number of leukocytes in the peripheral blood with a haematology analyzer (ERMA, Tokyo, Japan). At the conclusion of the experiment, each mouse was killed with an overdose of anaesthesia.

The retina was carefully removed, and flat-mounts were prepared using fluorescence anti-fading medium (Vector Laboratories, Burlingame, California, USA). The retinas were then examined using fluorescence microscopy (FITC filter; Olympus Optical, Tokyo, Japan) and the numbers of fluorescent dots in the retina within four separate circles of $800 \mu \mathrm{m}$ diameter next to the optic disc were counted. The average of the numbers within the four circles was considered the number of leukocytes migrated in the retina for each mouse.

\section{Statistical analysis}

All values were expressed as mean (SEM). The data were analyzed by repeated-measures analysis of variance, with post hoc comparisons tested with the Fisher protected least significant difference procedure. Unpaired t tests were used to compare results between groups at matched follow-up timepoints after lipopolysaccharide injection. Differences were considered significant when probability values were less than 0.05 .

\section{RESULTS}

\section{Physiological data}

Table 1 shows changes in physiological variables at various timepoints after lipopolysaccharide injection. There were no significant differences between the normal group and the iNOS ${ }^{-}$group in peripheral leukocyte count, mean arterial blood pressure or heart rate.

\section{Vessel diameters}

Fig $1 \mathrm{~A}$ and $\mathrm{B}$ show characteristic fundus images of a control mouse and a mouse $48 \mathrm{~h}$ after lipopolysaccharide injection, respectively. Fig 1C indicates changes in major retinal vessel 
Figure 1 Digitised monochromatic images of major retinal vessels obtained with a scanning laser ophthalmoscope in a control mouse $(A)$ and a mouse $48 \mathrm{~h}$ after lipopolysaccharide (LPS) injection (B). Time course of the diameters of major retinal arteries and veins after lipopolysaccharide injection (C). Values are mean (SEM). $n=6$ at each timepoint for each group. ${ }^{*} \mathrm{p}<0.05$ compared with artery or vein of normal mice. iNOS, inducible nitric oxide synthase.
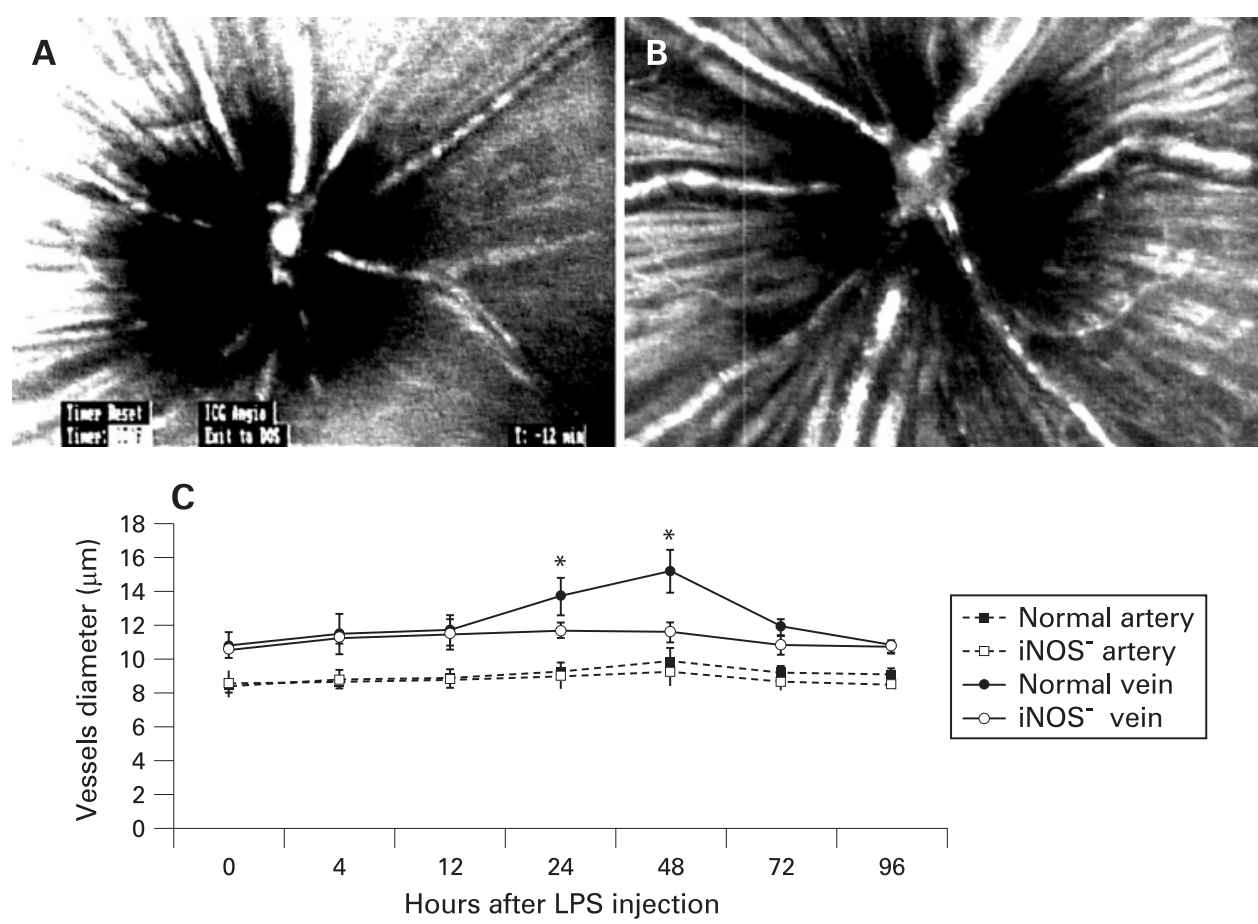

- Normal artery $\rightarrow-$ iNOS $^{-}$artery

- Normal vein - iNOS- vein

Hours after LPS injection

along major retinal veins but not along any major retinal arteries. At $4 \mathrm{~h}$ after lipopolysaccharide injection, several leukocytes were observed rolling along the venous walls. The flux of rolling leukocytes then increased gradually and peaked at $48 \mathrm{~h}$ after lipopolysaccharide injection, but this flux of rolling leukocytes decreased to almost basal levels at $96 \mathrm{~h}$ after lipopolysaccharide injection. In the NOS $^{-}$group, leukocyte rolling was significantly inhibited (fig $2, \mathrm{p}<0.001$ ). The maximal numbers of rolling leukocytes in the iNOS ${ }^{-}$group were reduced by $98.2 \%(p<0.001)$ at $48 \mathrm{~h}$ after lipopolysaccharide injection compared with those in the normal group.

\section{Number of migrated leukocytes}

At 30 minutes after $\mathrm{AO}$ injection, leukocytes in the vessels, as well as endothelial cells, became faint because of the washout effect but migrated leukocytes in the retina could still be identified as distinct fluorescent dots with the highest contrast. Accordingly, we observed only those leukocytes that were exposed to a high concentration of $\mathrm{AO}$ in the vessels for a few minutes and that were extravasated before the washout effect. Fig 4 shows the numbers of leukocytes that migrated in the retina at each timepoint. Few leukocytes were found in control retina. At $4 \mathrm{~h}$ after lipopolysaccharide injection, the flux of migrated leukocytes began to increase and peaked at $48 \mathrm{~h}$. In the iNOS $^{-}$group, leukocyte migration was significantly inhibited $(p<0.001)$; the maximal numbers of migrated leukocytes in the iNOS $^{-}$group was reduced by $74.0 \%(p<0.001)$ at $24 \mathrm{~h}$ compared with migrated leukocytes in the normal mouse group (fig 3 and fig 4).

\section{DISCUSSION}

In mouse retina with EIU, we demonstrated that a lack of iNOS attenuated leukocyte rolling along the major retinal veins and subsequent leukocyte migration into the retina. Moreover, in EIU mice, the diameters of major retinal arteries and veins of iNOS knockout mice were smaller than those of normal mice at all timepoints studied. On the basis of these findings, we
Figure 2 Time course of the flux of rolling leukocytes along major retinal veins after lipopolysaccharide (LPS) injection. Values are mean (SEM). $n=6$ at each timepoint for each group. ${ }^{*} \mathrm{p}<0.05$ compared with normal mice. iNOS, inducible nitric oxide synthase. 
Figure 3 Migrated leukocytes in the retina were observed as fluorescent dots (arrows) at 30 minutes after acridine orange injection. A significant reduction in leukocyte migration was seen in the inducible nitric oxide synthase (iNOS) knockout mouse $(A)$ compared with that of a normal mouse (B) at $48 \mathrm{~h}$ after lipopolysaccharide injection.
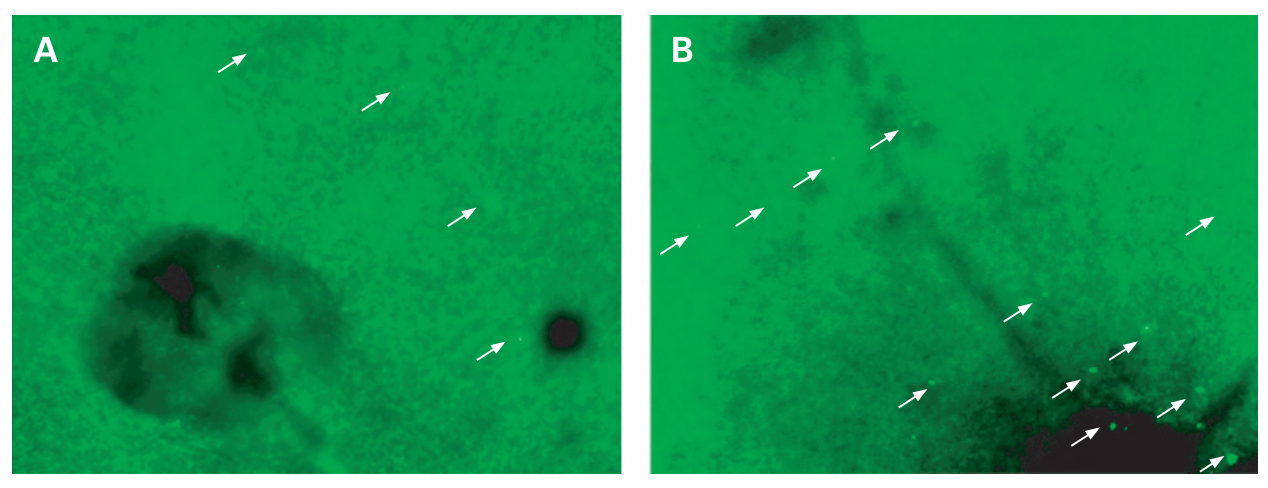

suggest that iNOS might stimulate inflammation by leukocyteendothelial cell interactions.

$\mathrm{NO}$ is a free radical produced in mammalian cells from Larginine and oxygen by isoforms of an enzyme known as NOS: neuronal NOS, iNOS and endothelial NOS. The role of NO in inflammation is an extremely controversial area of research, with studies demonstrating both beneficial and deteterious effects after NOS inhibition..$^{20}$ Mandai et a ${ }^{11}$ demonstrated that the expression of iNOS is upregulated during EIU and apparently plays an important role in the pathogenesis of EIU. ${ }^{11}$ The authors also noted that NO derived from iNOS regulates the expression of P-selectin and ICAM-1 and contributes to vasodilation. Also, Sennlaub et $a^{21}$ reported that iNOS mediates retinal apoptosis in ischaemic proliferative retinopathy. In contrast, although the exact mechanisms are not fully understood, other groups have demonstrated that $\mathrm{NO}$, released by either endothelial NOS or iNOS, modulates the leukocyte-endothelial cell interaction. Dal Secco et a ${ }^{22}$ reporterd that selective inhibitors of iNOS and endothelial NOS increase leukocyte rolling and adhesion to endothelial cells and increase neutrophil transmigration to sites of inflammation. In addition, Charlotte et $a^{23}$ demonstrated that iNOS expressed in the outer retina might play a protective role in experimental autoimmune uveitis. In the current study, leukocyte rolling and migration in the immunosuppressed iNOS $^{-/-}$mouse retina were dramatically suppressed compared with the normal mouse retina after lipopolysaccharide injection. These dichotomous results might be because the biological effects of iNOS may be derived from various inflammatory processes at the level of the microcirculation.

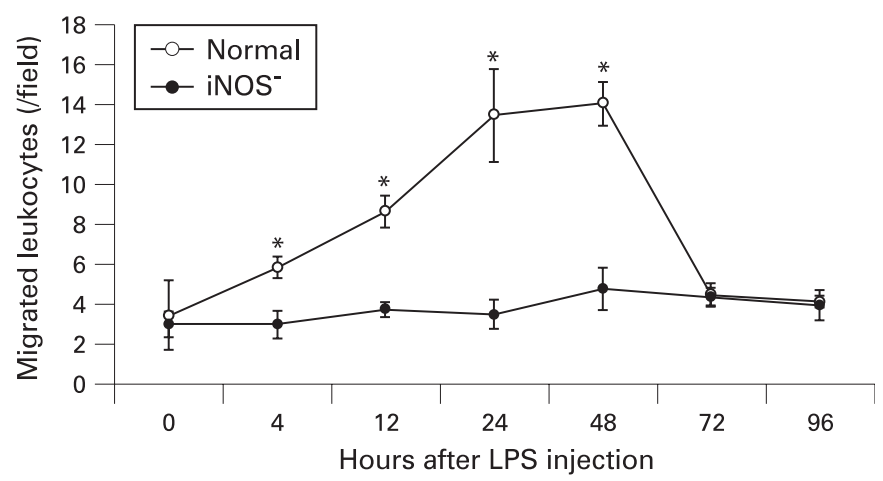

Figure 4 Time course of the number of leukocytes accumulating in the retinal microcirculation after lipopolysaccharide (LPS) injection. Values are mean (SEM). $n=6$ at each timepoint for each group. ${ }^{*} p<0.05$ compared with normal mice. iNOS, inducible nitric oxide synthase.
Rosenbaum et $a^{24}$ reported that leukocyte-endothelial dynamics were not the same in different vascular beds (iris, limbus, choroid) in the mouse eye and that the inflammatory response differed between these vascular beds. Our findings support their report in the aspect of the difference in status of microcirculation in the retina with EIU from that in normal retina and that in other vascular beds with EIU. Also, Becker et $a^{25}$ demonstrated that deletion of the IL- 8 receptor homologue reduced leukocyte migration but had minimal effects on leukocyte rolling with the use of iris rhodamine angiography. We think IL-8 might trigger leukocyte migration rather than rolling and iNOS might have suppressive effects on both leukocyte rolling and migration through regulation of $\mathrm{P}$-selectin and ICAM-1.

In the present study, the diameters of major retinal arteries and veins of the iNOS $^{-}$group were smaller than those of the normal group at all times. In arteries of the NOS $^{-}$group, slight vasoconstriction was seen after lipopolysaccharide injection, whereas the veins showed vasoconstriction compared with the normal group. In general, lipopolysaccharide-induced EIU causes significant vasodilation. ${ }^{26}$ It was previously demonstrated that diabetes reduced vein vasodilation in the retinas of rats with EIU, probably by the decreased expression of iNOS. ${ }^{27}$ This decreased expression of iNOS would also explain the suppressed leukocyte rolling and accumulation, because of its effect on the expression of P-selectin and ICAM-1. ${ }^{12-14}$ Our findings are consistent with these preceding reports.

In conclusion, we have demonstrated that a lack of iNOS attenuates leukocyte rolling along major retinal veins and the subsequent migration of leukocytes into the retinas of mice with EIU. To our knowledge, this study is the first to show that a lack of iNOS suppresses leukocyte-endothelial cell interactions in EIU of mouse retina. This suggests that iNOS may play a role in the management of patients with uveitis and other ocular inflammatory conditions.

Funding: This work was supported by a grant from the Japan National Society for the Prevention of Blindness and by a grant-in-aid for scientific research from the Japan Society for the Promotion of Science.

Competing interests: None declared.

\section{REFERENCES}

1. Sugama $\mathbf{Y}$, Tiruppathi $\mathrm{C}$, offakidevi $\mathrm{K}$, et al. Thrombin-induced expression of endothelial P-selectin and intercellular adhesion molecule-1: a mechanism for stabilizing neutrophil adhesion. J Cell Biol 1992;119:935-44.

2. Sluiter W, Pietersma A, Lamers JM, et al. Leukocyte adhesion molecules on the vascular endothelium: their role in the pathogenesis of cardiovascular disease and the mechanisms underlying their expression. J Cardiovasc Pharmacol 1993;22(Suppl 4):S37-44.

3. Ghezzi P, Dinarello CA, Bianchi M, et al. Hypoxia increases production of interleukin-1 and tumor necrosis factor by human mononuclear cells. Cytokine 1991;3:189-94. 
4. Matsuo Y, Kihara T, Ikeda M, et al. Role of neutrophils in radical production during ischemia and reperfusion of the rat brain: effect of neutrophil depletion on extracellular ascorbyl radical formation. J Cereb Blood Flow Metab 1995;15:941-7.

5. Prendergast RA, lliff CE, Coskuncan NM, et al. T cell traffic and the inflammatory response in experimental autoimmune uveoretinitis. Invest Ophthalmol Vis Sci 1998;39:754-62.

6. Liversidge J, Sewell HF, Forrester JV. Interactions between lymphocytes and cells of the blood-retina barrier: mechanisms of T lymphocyte adhesion to human retinal capillary endothelial cells and retinal pigment epithelial cells in vitro. Immunology 1990; 71:390-6.

7. Yoshida M, Yoshimura N, Hangai M, et al. Interleukin-1 alpha, interleukin-1 beta, and tumor necrosis factor gene expression in endotoxin-induced uveitis. Invest Ophthalmol Vis Sci 1994;35:1107-13.

8. Rosenbaum JT, McDevitt HO, Guss RB, et al. Endotoxin-induced uveitis in rats as a model for human disease. Nature 1980;286:611-13.

9. Bhattacherjee $\mathbf{P}$, Williams RN, Eakins KE. An evaluation of ocular inflammation following the injection of bacterial endotoxin into the rat foot pad. Invest Ophthalmol Vis Sci 1983:24:196-202

10. Miyamoto K, Ogura $Y$, Hamada $\mathrm{M}$, et al. In vivo quantification of leukocyte behavior in the retina during endotoxin-induced uveitis. Invest Ophthalmol Vis Sci 1996:37:2708-715

11. Mandai M, Yoshimura N, Yoshida M, et al. The role of nitric oxide synthase in endotoxin-induced uveitis: effects of NG-nitro L-arginine. Invest Ophthalmol Vis Sci 1994;35:3673-80.

12. Lush CW, Cepinskas G, Sibbald WJ, et al. Endothelial E- and P-selectin expression in iNOS-deficient mice exposed to polymicrobial sepsis. Am J Physiol Gastrointest Liver Physiol 2001;280:G291-7.

13. Cuzzocrea S, Chatterjee PK, Mazzon E, et al. Role of induced nitric oxide in the initiation of the inflammatory response after postischemic injury. Shock 2002;18:16976.

14. Hierholzer C, Kalff JC, Billiar TR, et al. Induced nitric oxide promotes intestinal inflammation following hemorrhagic shock. Am J Physiol Gastrointest Liver Physiol 2004:286:G225-33.
15. Thillaye-Goldenberg B, Goureau O, Naud MC, et al. Delayed onset and decreased severity of experimental autoimmune uveoretinitis in mice lacking nitric oxide synthase type 2. J Neuroimmunol 2000;110:31-44.

16. Liversidge J, Dick A, Gordon S. Nitric oxide mediates apoptosis through formation of peroxynitrite and Fas/Fas-ligand interactions in experimental autoimmune uveitis. Am J Pathol 2002;160:905-16.

17. Rajendram R, Saraswathy S, Rao NA. Photoreceptor mitochondrial oxidative stress in early experimental autoimmune uveoretinitis. Br J Ophthalmol 2007;91:531-7.

18. Miyahara S, Kiryu J, Miyamoto K, et al. In vivo three-dimensional evaluation of leukocyte behavior in retinal microcirculation of mice. Invest Ophthalmol Vis Sci 2004; 45:4197-201

19. Wei X0, Charles IG, Smith A, et al. Alterrd immune responses in mice lacking inducible nitric oxide synthase. Nature 1995;375:408-11.

20. Hickey MJ, Sharkey KA, Sihota EG, et al. Inducible nitric oxide synthase-deficient mice have enhanced leukocyte-endothelium interactions in endotoxemia. Faseb $J$ 1997;11:955-64.

21. Sennlaub F, Courtois $Y$, Goureau 0 . Inducible nitric oxide synthase mediates retinal apoptosis in ischemic proliferative retinopathy. J Neurosci 2002:22:3987-93.

22. Dal Secco D, Moreira AP, Freitas A, et al. Nitric oxide inhibits neutrophil migration by a mechanism dependent on ICAM-1: role of soluble guanylate cyclase. Nitric Oxide 2006;15:77-86.

23. Charlotte F, Ito S, Wu G, et al. Highly selective inhibitor of inducible nitric oxide synthase enhances S-antigen-induced uveitis. Curr Eye Res 2003:26:1-7.

24. Rosenbaum JT, Brischetto M, Crespo $\mathrm{S}$, et al. Simultaneous in vivo imaging of leukocyte migration: heterogeneity among iris, limbus, and choroid vessels. Curr Eye Res 2002:24:214-18.

25. Becker MD, O'Rourke LM, Blackman WS, et al. Reduced leukocyte migration, but normal rolling and arrest, in interleukin-8 receptor homologue knockout mice. Invest Ophthalmol Vis Sci 2000;41:1812-17.

26. Miyamoto K, Ogura Y, Hamada M, et al. In vivo neutralization of P-selectin inhibits leukocyte-endothelial interactions in retinal microcirculation during ocular inflammation. Microvasc Res 1998:55:230-40.

27. Tamura H, Kiryu J, Miyamoto K, et al. In vivo evaluation of ocular inflammatory responses in experimental diabetes. Br J Ophthalmol 2005;89:1052-7. 